Recovery as a concept has gained increased attention in the field of mental health. There is an expectation that service providers use a recovery framework in their work. This raises the question of what recovery means, and how it is conceptualised and operationalised. It is proposed that service providers approach the application of recovery principles by considering systematically individual recovery goals in multiple domains, encompassing clinical recovery, personal recovery, social recovery and functional recovery.

This approach enables practitioners to focus on service users' personal recovery goals while considering parallel goals in the clinical, social, and role-functioning domains. Practitioners can reconceptualise recovery as involving more than symptom remission, and interventions can be tailored to aspects of recovery of importance to service users.

In order to accomplish this shift, practitioners will require effective assessments, access to optimal treatment and care, and the capacity to conduct recovery planning in collaboration with service users and their families and carers. Mental health managers can help by fostering an organisational culture of service provision that supports a broader focus than that on clinical recovery alone, extending to client-centred recovery planning in multiple recovery domains.

\title{
Conceptualising Recovery in Mental Health Rehabilitation
}

\author{
Chris Lloyd, ${ }^{1}$ Geoff Waghorn ${ }^{1}$ and Philip Lee Williams ${ }^{2}$
}

\section{Introduction}

In mental health, the term 'recovery' is commonly used to refer to the lived experience of the person coming to terms with, and overcoming the challenges associated with, having a mental illness (Shepherd et al 2008). The term 'recovery' has evolved as having a special meaning for mental health service users (Andresen et al 2003) and consistently refers to their personal experiences and expectations for recovery (Slade et al 2008). On the other hand, mental health service providers often refer to a 'recovery' framework in order to promote their service (Meehan et al 2008). However, practitioners lean towards a different meaning-in-use,

\footnotetext{
${ }^{1}$ The University of Queensland, Australia.

${ }^{2}$ St Vincent's University Hospital, Elm Park, Dublin, Ireland.
}

Corresponding author: Dr Chris Lloyd, Senior Lecturer, Queensland Centre for Mental Health Research and the School of Population Health, The University of Queensland, Locked Bag 500, Sumner Park BC, QLD 4074, Australia. Email: Lloyd@onthenet.com.au

Submitted: 18 December 2007.

Accepted: 21 July 2008.

Key words: Recovery, mental health, occupational therapy, service orientation. Reference: Lloyd C, Waghorn G, Williams PL (2008) Conceptualising recovery in mental health rehabilitation. British Journal of Occupational Therapy, 71(8), 321-328. which is better described as 'clinical recovery' and is measured routinely in terms of symptom profiles, health service utilisation, health outcomes and global assessments of functioning. These very different meanings-in-use of the same term have the potential to cause considerable confusion to readers of the mental health literature. Researchers have recently identified an urgent need to clarify the recovery concept so that a common meaning can be established and the construct can be defined operationally (Meehan et al 2008,

Slade et al 2008).

This paper aims to delineate a construct of recovery that can be applied operationally and consistently in mental health. The criteria were twofold:

1. The dimensions need to have a parsimonious and near mutually exclusive internal structure

2. All stakeholder perspectives and interests, including those of the wider community, need to be accommodated.

With these criteria in mind, the literature was revisited to identify possible domains. It was subsequently identified that the recovery literature can be reclassified into components that accommodate the views of service users, practitioners, rehabilitation providers, family and carers, and the wider community. The recovery dimensions identified were clinical recovery, personal recovery, social recovery and functional recovery. 


\section{Recovery}

One of the earliest and most influential descriptions of recovery came out of the consumer movement. Deegan (1988) defined recovery as both a process and an outcome:

Recovery is a process, a way of life, an attitude, and a way of approaching the day's challenges. It is not a perfectly linear process. At times our course is erratic and we falter, slide back, regroup again ... The need is to meet the challenge of the disability and to re-establish a new and valued sense of integrity and purpose within and beyond the limits of the disability; the aspiration is to live, work, and love in a community in which one makes a significant contribution (p15).

Another influential definition was proposed by a leading mental health service provider and researcher, Anthony (1993):

... a deeply personal, unique process of changing one's attitudes, values, feelings, goals, skills, and/or roles. It is a way of living a satisfying, hopeful and contributing life even with limitations caused by illness. Recovery involves the development of new meaning and purpose in one's life as one grows beyond the catastrophic effects of mental illness (p21).

\section{Personal themes of recovery}

The personal themes of hope, empowerment and meaningful life activity are consistently identified in the recovery literature (Torrey et al 2005, Lehman 2006, Shepherd et al 2008). These themes have also been identified empirically (Andresen et al 2003). They have been subsequently adopted by leading mental health services as implicit standards for service user orientated and family-centred services. These are intended to address directly the goals and assistance needs of the service user and family, rather than continuing simply to meet the needs of service providers. These implicit standards convey the expectation that there should be a focus on optimal treatment and evidence-based services that promote service users' capacity to pursue their lives in their community to the fullest extent possible, while embracing the personal process of recovery (Lehman 2006).

The personal view of recovery departs from the concept of clinical recovery, which implicitly assumes that, in the majority of cases, correct assessment and optimal treatment are sufficient to achieve full symptom remission. This, in turn, enables people to return, mostly without further assistance, to premorbid levels of community functioning. An exclusive focus on clinical recovery is a traditional priority of mental health services, especially when resources are limited (Meehan et al 2008). However, this is a view of recovery not shared by users of mental health services (Shepherd et al 2008) and is also not shared by practitioners of evidence-based supported employment programmes (Lloyd and Waghorn 2007).

\section{Delineation of mental health recovery}

Without further delineation of the mental health recovery construct, there is a danger that developments in the recovery literature will not transfer to substantial change in the way that mental health services are delivered. Several strategies have been recommended to address this issue. Slade et al (2008) suggested distinguishing personal from clinical recovery goals. Meehan et al (2008) encouraged the involvement of the wider community in a debate to define the limits of recovery-based mental health services. Liberman et al (2008) suggested defining recovery in terms of individual improvements in specific life domains, rather than using a global index of functioning or indices of general recovery. They considered that an emphasis on measuring improvement in specific life areas would facilitate clinicians communicating more clearly with service users about the goals of treatment and psychosocial rehabilitation.

Since recovery principles are now widely promoted by both service users and mental health service providers, there is an urgent need to elucidate this rhetoric in order to understand what is meant by the term 'recovery', and how this can be operationalised into daily practice by occupational therapists and other allied health professionals. Adding a recovery framework to treatment and rehabilitation perspectives is intended to accommodate service user aspirations that hope, respect and pathways to community participation are incorporated into the daily activities of psychiatric rehabilitation. Importantly, this requires reframing the treatment enterprise from the therapist's perspective to the person's perspective. This has implications for how mental health treatment, care and rehabilitation are conceptualised and delivered, and the degree to which such care is acceptable to, and effective for, the people participating in mental health rehabilitation (Davidson et al 2006).

\section{Rehabilitation}

'Rehabilitation' is also a term that needs to be differentiated from recovery. Mental health professionals and rehabilitation professionals typically plan rehabilitation activities that involve targeted interventions to assist people to acquire and apply new skills, utilise supports and access the resources required to live a meaningful life in the community of their choice (Mitchell 2003). Service users, on the other hand, rarely mention rehabilitation, but instead talk about 'recovery' as a complex, non-linear and multidimensional personal process of getting well and getting on with their lives (Andresen et al 2003). Mental health professionals similarly consider rehabilitation to be multidimensional and often claim that rehabilitation services are also provided within a 'recovery' framework, perhaps in order to maintain congruence with the now widely accepted service user perspectives on recovery.

\section{Recovery-oriented evidence-based programmes}

Recovery from severe mental illness has been seen as a legitimate vision to guide mental health practice and 
policy (Anthony 2003). Although recovery is a deeply personal, unique journey, the external environment, including the service providing treatment and care, has an impact on the course of this journey. Farkas et al (2005) identified four key recovery values that should guide recovery-oriented evidence-based programmes. The service should first focus on the individual, acknowledging his or her strengths, talents and interests as well as his or her limitations. There should then be a focus on people's rights to full partnership in all aspects of their recovery. The service can also encourage people's rights to make choices regarding their own desired goals and outcomes. Lastly, there is a belief in the inherent capacity of any individual to recover (Farkas et al 2005). Ridgway (2001) described recovery as a journey that is affected by a complex array of factors. The themes from the accounts of recovery from prolonged psychiatric disability are outlined in Table 1.

\section{Table 1. Themes from personal accounts of recovery} (Ridgway 2001)

\footnotetext{
- The reawakening of hope after despair

- Breaking through denial and achieving understanding and acceptance

- Moving from withdrawal to engagement and active participation in life

- Active coping rather than passive adjustment

- No longer viewing oneself primarily as a person with a psychiatric disorder and reclaiming a positive sense of self

- Moving from alienation to a sense of meaning and purpose

- A complex and nonlinear journey

- The journey involves support and partnership
}

The literature advocates for mental health care change because it has been found that professional behaviour has the potential either to help or to hinder consumer recovery (Torrey et al 2005). Torrey and Wyzik (2000) suggested that embracing a recovery vision is not easy for most practitioners. This difficulty results from the concept being poorly defined and not operationalised. Many practitioners carry a large caseload and concentrate on high priorities, which may or may not involve evidence-based practices. In many instances, service users continue to receive services that have never been scrutinised scientifically (Liberman et al 2008). However, service delivery improvement efforts can be informed directly by the lessons learned from the lived experiences of people with a mental illness.

\section{Opportunities for recovery}

Creating opportunities for recovery ought to be a core mission of mental health services. The service orientation is an important factor in facilitating the engagement of service users in the recovery journey. Services can support this process by working with, and not on, service users in order to address life issues, including, but not limited to, symptom management. Torrey and Wyzik (2000) suggested that it is important that services communicate hope; help service users to develop the skills and knowledge that they need to take personal responsibility for their health; and support service users' efforts to get on with life beyond illness.
A constant theme in service orientation is the importance of assisting service users to continue to grow at all points in their recovery journey. The service planning process is a natural focus for recovery-oriented quality improvement efforts. It has been suggested that mental health services can help service users to participate actively in the management of their health concerns through offering treatment choices and providing the information, skills and supports that service users need to be able to choose wisely (Torrey and Wyzik 2000). It is only by having shared decision making with service users that they are able to exercise their power and make their treatment relevant to their individual interests and needs (Torrey and Wyzik 2000).

\section{Clinical recovery}

Severe mental illness, such as schizophrenia, has a profound impact on the person above and beyond the symptoms, including a loss of self-esteem, alienation from friends and family, interruption of school and career, and the experience of stigma. The longer and more severe the course of the illness, the greater the impact that these changes are likely to have on a person's life (Bellack 2006). Symptom remission alone is inadequate for a definition of recovery from schizophrenia. The dimensions of improved psychosocial functioning are also integral to the concept of recovery. These dimensions include work, school, family life, recreation and independent living (Liberman and Kopelowicz 2005).

A number of barriers to recovery were identified by Smith (2000). These included stigma, symptoms, the lack of financial resources, a limited access to services and occasional eruptive responses to life pressures. Recovery involves much more than learning to cope physically with the disability. It involves learning new ways to perceive and interact with the environment and acquiring new skills to negotiate environmental elements, such as a system of service providers, family members and friends (Smith 2000). Recovery or living with any chronic health condition requires individuals to learn how to manage their illness in collaboration with treatment providers.

It was suggested that the various psychological interventions for helping people to manage their symptoms and prevent relapses needed to be consolidated into a single standard programme (Mueser et al 2006). This includes psychoeducation about mental illness and its treatments; cognitive-behavioural approaches to encourage medication adherence; developing a relapse prevention plan; strengthening social support by social skills training; and coping skills training for the management of persistent residual symptoms.

\section{Personal recovery}

A service user oriented definition of psychological recovery has been described as 'the establishment of a fulfilling, 
meaningful life and a positive sense of identity founded on hopefulness and self-determination' (Andresen et al 2003, p588). This definition describes recovery from the psychological trauma and consequences of the illness rather than a cure or an absence of symptoms. The authors identified four component processes of recovery, which were hope, self-identity, meaning in life and responsibility. The re-establishment of important goals is a common theme among service user accounts of recovery, and the previous four components can be linked together by the addition of service user choice in the pursuit of personal goals.

Sells et al (2006) stated that community arenas appear to promote recovery to the extent that they foster both being with others who are supportive or receptive to support and doing things that feel meaningful, ultimately kindling and, over time, building a favourable redefinition of self. They analysed 12 narratives of people with a serious mental illness who considered themselves to be either recovered or in recovery in order to determine the benefits of spending time within various community settings. The key themes that emerged were the importance of understanding and acceptance by others; fun and enjoyment; role shifting (transformational quality of activity); meaningful routines (helpful in reducing stress and loneliness and fostering recovery); employment; spirituality; self-esteem; anger; and integrative aspects (a sense of affirmation of who they felt they were, apart from having a mental illness). Sells et al (2006) suggested that the findings appeared to support the notion that time spent in various community arenas could foster recovery.

Resnick et al (2005) studied recovery orientation among 1076 participants. They proposed four domains as an initial approach to the conceptualisation of recovery orientation. These domains were the capacity to feel empowered in one's life; self-perceptions of knowledge about mental illness and available treatments; satisfaction with quality of life; and hope and optimism for the future. The results indicated that promoting self-esteem and helping individuals to discover and attain their goals were beneficial to a recovery orientation. The authors also suggested that interventions for people with a severe mental illness should seek to promote the development of hope and optimism. Knowledge about the illness, the range of available treatments and the ways to navigate the system formed another area that Resnick et al (2005) identified as being important. Interventions should also focus on improving communication with family members, and with assisting service users to attain housing stability.

Mead and Copeland (2000) used their own experiences to explain the meaning and significance of recovery. The key facets of recovery identified included hope, personal responsibility for wellness, self-education, self-advocacy and peer support. They recommended that professionals address the learned helplessness of people who are stuck in the system by asking for their ideas about who they would like to become, and the supports that they wanted or needed to achieve this. Mead and Copeland (2000) suggested that in a recovery-based environment, more effort needs to be directed to facilitating choice and self-responsibility around behaviour.

Tooth et al (2003) investigated service user perspectives on recovery from schizophrenia among 57 people who identified themselves to be in recovery. The role of self was the most frequently reported theme. The person's determination to get better; his or her optimism; the acknowledgement of the need to take responsibility and help himself or herself; and understanding, managing and accepting the illness were the most frequently reported subcategories. The authors concluded that it was time to refocus on service users' stories and to give legitimacy to their experience.

Smith (2000) analysed the personal recovery stories of 10 people with a serious mental illness. The participants discussed various ways to maintain their recovery and their stories yielded 10 major strategies: accept your disability; believe in recovery; ensure that you are stabilised; accept your own responsibility for the disability; establish a structure for your daily life; seek support; take care of yourself; keep active; educate yourself; and protect your recovery. Smith (2000) noted that the familiar themes that emerged provide information for enhancing and speeding the recovery of people with a mental illness. The author highlighted the realisation that each person reaches the beginning point of recovery at a different pace, emphasising that it is important that practitioners help service users by directing them to community resources useful in their recovery. Smith (2000) added that practitioners need to remain encouraged and to give service users dedicated support in order to assist them on their recovery journey.

\section{Social recovery}

Recovery has been described as both an outcome and an ongoing process. Corrigan and Phelan (2004) studied the relationship between objective and subjective measures of social support with recovery among 176 participants. They found that both objective and subjective measures of social support were significantly associated with a process perspective of recovery. People with a mental illness who report larger numbers of people in, or more general satisfaction with, their social networks were likely to report such aspects of recovery as greater hope and being more oriented towards goals and success. The size and satisfaction with one's support network was not significantly associated with psychiatric symptoms. This study showed that recovery as a process involving hope and goal orientation is associated with social support. The authors suggested that these results support interventions that facilitate social networks, which include supported employment, housing and education, as well as family-focused services.

Secker et al (2002) explored the experiences of employment support as reported by 17 service users. 
Based on this small study, they suggested that a social recovery model offered a more promising way forward for supported employment than a clinical model of recovery. As expected, retaining a job emerged as more challenging than finding it in the first place. Ensuring the best possible job match to a person's preferences, and then negotiating service users' needs to be met on an ongoing basis as skills and confidence developed, emerged as an important factor in job retention. Follow-up support appeared critical in assisting people to remain in their jobs. This study suggests that a social recovery approach can contribute to providing more effective post-employment support.

For many people, social interaction is an important part of the recovery process. In a study conducted by Topor et al (2006), all 12 participants described themselves as being involved in relationships with others. These relationships were reported to play a major role in the course of their illness, and in their recovery. The contributions of family and longtime friends were evident in terms of standing alongside the person, being there for the person in recovery, and moving on with the recovery. Professionals were also found to contribute to recovery by conveying authoritative information to the person by virtue of their expert status, or by serving as intermediaries for various interventions that involved money, activities, groups, housing and socialising. Supportive professionals often did more than their formal role required and frequently did something different from what the person had come to expect from professionals. Throughout the narratives, participants talked about the importance of having support while they learned to cope with their illness.

Social contexts can exert a strong influence on a person's sense of self. Many people can, during the course of their mental illness, internalise societal notions of severe mental illness as they take on a patient's role and identity, becoming more lonely and isolated and more susceptible to internalised stigma (Mezzina et al 2006b). During processes of recovery and empowerment, the opportunities for social integration, like many forms of social activities, are described as ways and means for exercising and developing capacities and regaining power and status. Stigma emerges as one of the most common and tangible barriers to socialising in people with a psychosis, as well as contributing to a feeling of being different. Being excluded from a normal social life has a profound effect on how people see themselves in their social role, and participants often reported feeling that they were locked into their role as mental patients.

Mezzina et al (2006b) found that people try to overcome and deal with stigma and labelling in several different ways. The most important step towards becoming connected to others seemed to be accepting and understanding that there was a problem to begin with. Once they began to accept and articulate the symptoms and fallout from their illness, they began to associate themselves more closely with others in the same situation as a way to access opportunities and make friends. Work often remained at the centre of participants' lives. Work provides financial support for participation in social activities, giving people a chance to interact with others. The sense of belonging to a place or a group often occurred within the context of work or a similar interest club, in which friendships are formed based on common interests (Mezzina et al 2006b).

The social nature of recovery suggests that community mental health services can give more attention to the issues of social inclusion, civil rights and the participation of people with a psychosis in community activities of their choice (Mezzina et al 2006a). Facilitating integration in this way requires the mental health service system to re-envision its role from that of a treatment and rehabilitation provider to that of a broader community resource, responsible for catalysing and expediting people's access to opportunities, arenas and resources.

\section{Functional recovery}

Symptom reduction is insufficient to promote the recovery of function and wellbeing (Lehman 2006). There is a need for treatments and services that enhance these outcomes. Belief that there is hope for a better life is a large part of the recovery orientation. For service providers, this translates to conveying positive messages about the person's abilities and willingness to make changes to reach his or her goals. It also means providing the person with information, skills and positive support to assist him or her in achieving these goals (Salyers and Macy 2005).

Woodside et al (2006) examined the factors contributing to vocational success in a small qualitative study. Eight people who were recovering from a psychosis were interviewed. The authors found that people who returned to work successfully following a psychotic episode engaged in self-assessing their own health, worked to maintain and improve their mental health, and felt connected to others at their place of work. They suggested that the participants' vocational story appeared to be embedded in their own individual recovery. Woodside et al (2006) highlighted the importance of listening to the story of where service users are positioned in their recovery journey. Supported employment is an area in which professionals can help individuals to clarify their goals and develop meaningful activity that promotes self-esteem (Bond et al 2001).

A significant number of participants identified their illness as a life-transforming experience. Although the experience of the illness is a time of turmoil, many people experience recovery as a transformative process in which the old self is let go and a new, more functional sense of self emerges (Tooth et al 2003). Family psychoeducation has been shown to reduce symptoms and improve functioning in individuals with schizophrenia and to reduce the burden on families (Glynn et al 2006). There are aspects of existing family interventions that resonate with a recovery outlook, including service user 
directed goal setting and problem focus, educating people about their own illness, and focusing on the whole person by assessing his or her quality of life and not simply considering symptom constellations or hospitalisations.

Lloyd and Waghorn (2007) highlighted the importance of vocation in recovery for young people with psychiatric disabilities. They identified a number of barriers to restoring career paths. A focus on evidence-based mental health and community-based care was suggested, encompassing support and education for families and carers, evidence-based open employment, and evidence-based supported education. Evidence-based supported employment and supported education services now provide highly individualised approaches, including zero exclusion of people who want assistance, an exclusive focus on personal preferences and goals, time-unlimited support, and services closely coordinated with treatment and care services. Both programmes require a focus on the strengths of the person in order to increase motivation, engagement and expectations for success. In order to facilitate vocational recovery, it is important that conversations about work and education are initiated as early as possible; that rapid intensive vocational assistance is provided; and that any skills training needed is offered as part of post-employment support (Lloyd and Waghorn 2007).

\section{Implications}

There has been a significant shift towards considering the concept of recovery in everyday clinical practice in a majority of mental health services. Although this has created a positive shift in focus in the clinical care provided to service users, recovery remains an ambiguous concept that is difficult to apply to policies or behaviours. It is clearly important that services work to create cultures within their services that support the values underlying the recovery concept, as described above. However, it is also important for service users and service providers to increase their understanding of the multiple dimensions of recovery in order to be able to apply recovery values more effectively in clinical care.

In addressing the different domains of recovery as outlined in this paper, services can assist service users to become 'healthy', as defined by the World Health Organisation (1986). By this definition, health is seen as a resource for everyday life, not the objective of living; it is a positive concept, emphasising social and personal resources as well as physical capacities. Achieving this outcome has several implications for service providers.

The objectives of occupational therapy can focus on improving the recovery of people with a mental illness to reduce their social and economic marginalisation by increasing participation in society, increasing social integration and increasing social inclusion. In terms of increasing recovery, it is necessary to consider systematically the areas of clinical, personal, social and functional recovery.

\section{Clinical recovery}

Clinical recovery requires appropriate access to optimal forms of evidence-based treatments and long-term continuing health care. Importantly, this requires access to appropriate mental health and physical health services. The divide between mental and physical health services remains an ongoing treatment issue. Reducing the potential risk for negative outcomes from relapse and adverse life events is an important focus of care. This can be achieved by facilitating the service user to engage his or her local resources, including family and carers, the local community and local health services, such as general practitioners, in all aspects of their care when this is appropriate.

\section{Personal recovery}

In order to achieve clinical recovery, it is also important to attempt to address directly individuals' personal recovery goals. Occupational therapists can start by asking people about these goals and by providing help in all the goal domains of interest to that person, in ways in which the person agrees would be helpful. Progress in other domains, such as vocation, can also be used to address personal recovery goals positively, such as personal empowerment, a sense of identity and purpose in life. Although personal recovery may also improve through effective treatment and care, it is important to consider how personal recovery goals can be advanced in parallel rather than in series with treatment.

\section{Social recovery}

Although social recovery is influenced by the personal recovery progress of the service user, this is a domain that can be considered independently. Even in the early stages of treatment, conversations about social recovery goals can be initiated. The person can be helped to identify and make optimal use of the resources in the immediate environment. If the person has vocational goals and progress is achieved, the focus of care can shift towards helping the person to make optimal use of the new social opportunities linked to having a valued job or course of study. Occupational therapists can provide assistance to associate with people and make new friends; offer training in communication skills within a particular context; and help to develop strategies for managing personal information, as well as for preventing and countering stigma and unfair discrimination. The expected outcomes of this process are social skills transfer and greater social inclusion in the wider community.

\section{Functional recovery}

From within traditional clinically focused mental health services, occupational therapists and other allied health professionals have often advocated for greater attention to functional recovery in real world socially valued role domains. A number of socially valued roles have been identified, including independent living (for example, home duties, self-care, independent travel and financial management). Personal rehabilitation and self-development, including voluntary work, personal development courses 
and other activities facilitate personal rehabilitation goals other than formal study and employment. In addition, there are socially valued caring roles for children and other adults. Formal education or vocational training and competitive employment are two other socially valued role domains that need to be considered when helping a person to identify his or her real world functional recovery goals.

\section{Social inclusion}

Social inclusion is an important aspect to consider when facilitating recovery across the domains described. Occupational therapists need to consider social inclusion as an intervention goal, as well as how it can be facilitated directly. Access to adequate and satisfying social and emotional support from others when needed; preventing further stigma experiences, avoiding negative social interactions and preventing unfair discrimination; and encouraging participation in the local neighbourhood community can all be promoted. The aim is to help people to accumulate positive social interactions with regular people in their local community, so that their only source of positive social interactions is not restricted to service users or health professionals.

\section{Assessment of need}

A systematic assessment of recovery goals and preferred forms of assistance is required to begin recovery planning. This is needed at the initial stage of engagement in a service in order to accommodate the multidimensional nature of recovery. The key ingredients include listening carefully to service users' stories of past experiences with recovery and initiating conversations about recovery goals in each of the domains described. The motivation for change within a particular domain, and priorities among domains, can be determined. Next, a strengths' audit can be used to identify strengths, confidence and preferences with respect to the core activities required within each domain for which assistance is being sought. Help to accept and adjust to limitations caused by illness and treatments can be used to foster a pragmatic recovery orientation in order to have a fulfilling life despite those limitations. From this basic yet essential information, a personally tailored recovery plan based on the chosen recovery domains can be developed.

\section{Conclusion}

Recovery is a highly individual and personal journey for service users. Focusing on recovery requires service providers to change their focus of care and treatment. It is contended that it is important that service providers address not only clinical recovery but also functional recovery, personal recovery and social recovery, in order to provide more effective client-centred treatment and care.

Clinical recovery refers to helping service users to identify personally meaningful treatment goals, and helping people to obtain optimal treatment and care, while learning how best to manage their illness and treatment in the process of achieving those goals. Personal recovery attends to the needs of service users when seeking to make sense of the experience of mental illness and how it affects their future and their self-identity. Social recovery refers to the social dimension of recovery, with goals to restore social skills and social inclusion in the wider community. Functional recovery refers to recovery in the role domains of interest to the person that are also valued by the wider community.

In order to provide a recovery-oriented service systematically, providers such as occupational therapists can develop an understanding of how to offer assistance within each of these areas. Health care managers can assist by influencing their organisational culture positively in order to support the full application of a recovery approach. A mental health service that addresses these recovery domains systematically is also likely to improve health care outcomes and to reduce the overall disease burden of mental illness on the person, his or her family and the wider community.

\section{References}

Andresen R, Oades L, Caputi P (2003) The experience of recovery from schizophrenia: towards an empirically-validated stage model. Australian and New Zealand Journal of Psychiatry, 37(5), 586-94. Anthony WA (1993) Recovery from mental illness: the guiding vision for the mental health service system in the 1990s. Psychiatric Rehabilitation Journal, 16, 11-23.

Anthony WA (2003) Expanding the evidence base in an era of recovery. Psychiatric Rehabilitation Journal, 27(1), 1-2.

Bellack A (2006) Scientific and consumer models of recovery in schizophrenia: concordance, contrast, and implications. Schizophrenia Bulletin, 32(3), 432-42.

Bond GR, Becker DR, Drake RE, Rapp CA, Meisler N, Lehman AF, Bell MD, Blyler CR (2001) Implementing supported employment as an evidence-based practice. Psychiatric Services, 52(3), 313-22.

Corrigan P, Phelan S (2004) Social support and recovery in people with serious mental illnesses. Community Mental Health Journal, 40(6), 513-23.

Davidson L, O'Connell M, Tondora J, Styron T, Kangas K (2006) The top ten concerns about recovery encountered in mental health system transformation. Psychiatric Services, 57(5), 649-45.

Deegan P (1988) Recovery: the lived experience of rehabilitation. Psychosocial Rehabilitation Journal, 11, 11-19.

Farkas M, Gagne C, Anthony W, Chamberlin J (2005) Implementing recovery oriented evidence based programs: identifying the critical dimensions. Community Mental Health Journal, 41(8), 141-58.

Glynn S, Cohen A, Dixon L, Niv N (2006) The potential impact of the recovery movement on family interventions for schizophrenia: opportunities and obstacles. Schizophrenia Bulletin, 32(3), 451-63.

Lehman A (2006) Promoting recovery: achieving the promise. Schizophrenia Bulletin, 32(3), 430-31.

Liberman RP, Kopelowicz A (2005) Recovery from schizophrenia: a concept in search of research. Psychiatric Services, 56(6), 735-42.

Liberman JA, Drake RE, Sederer LI, Belger A, Keefe R, Perkins D, Stroup S (2008) Science and recovery in schizophrenia. Psychiatric Services, 59(5), 487-96.

Lloyd C, Waghorn G (2007) The importance of vocation in recovery for young people with psychiatric disabilities. British Journal of Occupational Therapy, 70(2), 50-59. 
Mead S, Copeland ME (2000) What recovery means to us: consumers' perspectives. Community Mental Health Journal, 36(3), 315-28.

Meehan TJ, King RJ, Beavis PH, Robinson JD (2008) Recovery-based practice: do we know what we mean or mean what we know? Australian and New Zealand Journal of Psychiatry, 42(3), 177-82.

Mezzina R, Davidson L, Borg M, Marin I, Topor A, Sells D (2006a) The social nature of recovery: discussion and implications for practice. American Journal of Psychiatric Rehabilitation, 9(1), 63-80.

Mezzina R, Borg M, Marin I, Sells D, Topor A, Davidson L (2006b) From participation to citizenship: how to regain a role, a status, and a life in the process of recovery. American Journal of Psychiatric Rehabilitation, 9(1), 39-61.

Mitchell S (2003) Rehabilitation and recovery. Auseinet, 19, November, 13-15.

Mueser K, Myer P, Penn D, Clancy R, Clancy D, Salyers M (2006) The illness management and recovery program: rationale, development, and preliminary findings. Schizophrenia Bulletin, 32(1), 32-43.

Resnick S, Fontana A, Lehman A, Rosenheck R (2005) An empirical conceptualization of the recovery orientation. Schizophrenia Research, 75(1), 119-28.

Ridgway P (2001) ReStorying psychiatric disability: learning from first person accounts of recovery. Psychiatric Rehabilitation Journal, 24(4), 335-43.

Salyers M, Macy V (2005) Recovery-oriented evidence-based practices: a commentary. Community Mental Health Journal, 41, 101-103.

Secker J, Membrey H, Grove B, Seebohm P (2002) Recovering from illness or recovering your life? Implications of clinical versus social models of recovery from mental problems for employment support services. Disability and Society, 17(4), 403-18.
Sells D, Borg, M, Marin I, Mezzina R, Topor A, Davidson L (2006) Arenas of recovery for persons with severe mental illness. American Journal of Psychiatric Rehabilitation, 9(1), 3-16.

Shepherd G, Boardman J, Slade M (2008) Making recovery a reality. London: Sainsbury Centre for Mental Health.

Slade M, Amering M, Oades L (2008) Recovery: an international perspective. Epidemiologia e Psichiatria Sociale, 17(2), 128-37.

Smith MK (2000) Recovery from a severe psychiatric disability: findings of a qualitative study. Psychiatric Rehabilitation Journal, 24(2), 149-58.

Tooth B, Kalyanasundaram V, Glover H, Momenzadah S (2003) Factors consumers identify as important to recovery from schizophrenia. Australasian Psychiatry, 11, Supplement 1, S70-77.

Topor A, Borg M, Mezzina R, Sells D, Marin I, Davidson L (2006) Others: the role of family, friends, and professionals in the recovery process. American Journal of Psychiatric Rehabilitation, 9(1), 17-37.

Torrey WC, Wyzik P (2000) The recovery vision as a service improvement guide for community mental health center providers. Community Mental Health Journal, 36(2), 209-16.

Torrey W, Rapp C, Van Tosh L, McNabb C, Ralph R (2005) Recovery principles and evidence-based practice: essential ingredients of service improvement. Community Mental Health Journal, 41(1), 91-100.

Woodside H, Schell L, Allison-Hedges J (2006) Listening for recovery: the vocational success of people living with mental illness. Canadian Journal of Occupational Therapy, 73(1), 36-43.

World Health Organisation (1986) The Ottawa Charter for Health Promotion. Available at: http://www.paho.org/English/AD/SDE/HS/ OttawaCharterEng.pdf Accessed on 20.08.07.

\section{Letters to the Editor}

\section{Sexuality and health care}

Madam,

We are writing in response to the recent editorial by Subhajit Sengupta and Brendon Stubbs on 'Sexuality and health care: can we carry on ignoring the issue?' (BJOT July 2008). As occupational therapists who co-facilitate workshops for people with learning disabilities to explore relationships and sexuality, we felt that the editorial was timely.

Although we agree that there is a lack of emphasis in undergraduate courses, we felt that the reason suggested for occupational therapists not exploring sexuality issues with other clients was a little simplistic.
Initially, therapists have to explore their own values, morals and beliefs in relation to sexuality before embarking on discussions with clients to ensure a non-judgemental approach. Often, religious or cultural beliefs can be a factor in occupational therapists' involvement in sexuality training or, indeed, the lack of it. Sexuality is a complex area, which involves deep reflections and self-exploration in order to ensure that one's own values and beliefs do not transfer during client interaction.

Depending on need and client group, sexual issues can be addressed at various levels, from handing over leaflets to facilitating groups. However, therapists need to find the level at which they feel competent and comfortable. Facilitating a group, for instance, may not be suitable for all therapists due to the level of personal disclosure regarding their own sexual journey.

We would, however, fully endorse the view that sexuality should be offered as an option on undergraduate or postgraduate courses, possibly in partnership with existing sexual health clinics and other areas of current expertise.

\section{Margaret Spencer,}

Co-facilitator, Relationships Matter, and Senior Lecturer in Occupational Therapy, Sheffield Hallam University.

\section{Chris Wainwright,}

Co-facilitator, Relationships Matter, and Occupational Therapist, W.O.R.K. Ltd, Sheffield.

Useful website: Relationships-matter.co.uk 